\title{
The Function of miR-31 Expression in Differentiation of Neural Stem Cells
}

\author{
Xiao Li1,2, Yuantao Gao3, Pengfei Li1,2, Feng Tian1,2, Jing Wang1,2, Chunfang Wang1,2* \\ ${ }^{1}$ Experimental Animal Center, Shanxi Medical University, Shanxi, China \\ ${ }^{2}$ Shanxi Key Laboratory of Laboratory Animal and Animal Model of Human Diseases, Shanxi Medical University, \\ Shanxi, China \\ ${ }^{3}$ Nanchang University Queen Mary School, Nanchang, China \\ Email: *wangchunfang1@163.com
}

Received 7 February 2016; accepted 10 March 2016; published 17 March 2016

\section{Abstract}

Spinal cord injury (SCI) is a severe complication of acute spinal injury, it can lead to axonal degeneration and necrosis of neurons, causing damage cross section below the movement, then loss of sensory, reflex and autonomous control function below the damage section. Nowadays, there is increasing evidence that transplantation of neural stem cells can help to repair spinal cord injury after spinal cord injury. The aim of this study was to research the function of miR-31 expression in differentiation of neural stem cells. Mir-31 was transfected the mimics and inhibitor into neural stem cells. By the morphological observation, the cell over expression of miR-31 was closer to NSCs. With the RT-PCR, Hb9 and STMN1 were up-regulate when miR-31 was inhibited, and Nestin was down-regulate. When miR-31 was over expression, the expression of Nestin and $\mathrm{Hb} 9$ was up-regulate. These findings suggest that miR-31 may play a significant role in proliferation and differentiation of neural stem cell and are potential targets for therapeutic interventions following spinal cord injury.

\section{Keywords}

Spinal Cord Injury, microRNA, Neural Stem Cells, Transplantation, Mouse

\section{Introduction}

There is a wide variety of reasons happened to spinal cord injury (SCI). The most common cause for spinal cord is incident trauma. The clinical treatment for SCI lacks effective and safe strategies which have become a severe socioeconomically issue. Recent researches have shown that nerve stem cells (NSCs) replacement is an effective strategy for the treatment of SCI. (Paul Lu et al., 2014; Suzuki SO et al., 2003) [1] [2]. How induce more NSCs to motor neuron cells (MNs) is the vital part.

MicroRNAs are a novel class of short, 18 - 25 nucleotide-long non-coding small RNAs. It play an important regulatory role in gene expression by binding to the 3'untranslated region of target mRNAs transcripts for de-

"Corresponding author.

How to cite this paper: Li, X., Gao, Y.T., Li, P.F., Tian, F., Wang, J. and Wang, C.F. (2016) The Function of miR-31 Expression in Differentiation of Neural Stem Cells. Journal of Biosciences and Medicines, 4, 57-64. 
gradation or inhibiting protein translation to determine a serial of vital life process such as cell differentiation, tissue repair and embryonic development. Individual miRNA can be inhibited multiple target genes simultaneously to regulated complex physiological and pathological process. In recent years, a lot of studies have confirmed microRNA may be involved in the regulation of differentiation in NSCs. (Maged M harrza, 2014; Chao Shi Niu, 2013; Eyal Mor, 2013) [3]-[5]. Therefore, it is very important to study how microRNAs regulated relevant signaling pathway to affect protein synthesis. Our previous study certify miR-31 is significant difference between NSCs and MNs which is highly expressed in NSCs and low expressed in MNs. (Hongen Wei, 2010) [6] It had been also reported to be an embryonic stem-specific microRNA. So we think that miR-31 may play an important role in maintaining NSCs in an undifferentiated state, but the concrete mechanism for miR-31 participate in is unclear. To directly address the role of miR-31 in neuronal development, miRNA Mimics and miRNA Mimics control are induced NSCs to observe the expression changes of those genes to analysis the signaling pathway of miR-31 regulated.

\section{Materials and Methods}

\subsection{Animals}

Embryonic day 14 FVB mouse were used. This study was performed with permission of the local animal use and care committee in accordance with the applicable and governmental regulations.

\subsection{Isolation, Culture and Identification of NSCs}

Spinal cords obtained from E14 mouse were collected in sterile D-Hank's balanced salt solution and immediately processed for tissue culture. Spinal cords were mechanically separated from the surrounding connective tissue using sterile instruments under a dissecting microscope. Then discarded spinal meninges and triturated surplus ages by pipette to dissociate cells. Dispersed cells ere centrifuged at $1000 \mathrm{rpm}$ for 10 min. the cells' pellet was resuspended in KnockOut ${ }^{\mathrm{TM}} \mathrm{DMEM} / \mathrm{F} 12$ supplemented with StemPro ${ }^{\circledR}$ Neural Supplement, EGF and FGFb (Bibco) and was plated in $25 \mathrm{~cm}^{2}$ Cell Culture Flask, and incubated in a $\mathrm{CO}_{2}$ Incubator of $5 \% \mathrm{CO}_{2}$ at $37^{\circ} \mathrm{C}$. The medium was changed for half dose every $3 \mathrm{~d}$.

The cells were observed every day. When the clonal globes were formatted detected by immunofluorescence. Cells culture were fixed in cold $4 \%$ paraformaldehyde and washed 3 times with PBS at $25^{\circ} \mathrm{C}$. Immunocytochemistry was carried out using standard protocols. Cell nuclei were counterstained with hochest 33342. The first antibodies was rabbit-anti-Nestin, 1:500. The second antibodies was goat-anti-rabbit IgG-FITC conjugate, 1:200. Nuclei were stained using Hochest 33258 via immunocytochemistry.

\subsection{Transfection}

To investigate the efficacy of RNAiMAX, the cells were seeded at $1 \times 10^{6}$ cells/well in 6 well plates, and after $4 \mathrm{~h}$ incubation at $37^{\circ} \mathrm{C}$ to adhere to the glass glide, the cells were transfected with $30 \mathrm{pM}$ siRNA. The transfection reagent was Lipofectamine RNAiMAX (Invitrogen) and Block-iTTM Alexa FluorR Red Lot No: 1477915 (invitrogen) were used according to the manufacturers' instructions. After $24 \mathrm{~h}, 48 \mathrm{~h}$ and $72 \mathrm{~h}$ incubation at $37^{\circ} \mathrm{C}$ the cells were observed by fluorescence microscope.

Cells were seeded at a density of $1 \times 10^{6}$ cells/well in 6 well plates. After $4 \mathrm{~h}$ incubation at $37^{\circ} \mathrm{C}$, during which time the cells had adhered to the plastic, the cells were transfected with $30 \mathrm{pM}$ siRNA. The transfection reagents were Lipofectamine ${ }^{\circledR}$ RNAiMAX, mmu-miR-31-5p mirVanaTM miRNA mimic, mirVanaTM miRNA mimic Negative Control, mmu-miR-31 Anti-miRTM miRNA inhibitor, Anti-miRTM Negative Control (Ambion). Operations were used according to the manufacturers' instructions. After $72 \mathrm{~h}$ incubation at $37^{\circ} \mathrm{C}$ the cells were used immunohistochemical method to detect ChAT expression in cells.

\subsection{Isolation of RNA for $q R T-P C R$}

After 72 h we isolated total RNA and analyzed by real-time PCR. Total RNA was extracted using the mirVana miRNA Isolation Kit (Ambion, Austin, TX, USA) according to the manufacturer's protocol. The purified RNA was quantified by determining the absorbance at $260 \mathrm{~nm}$ using an UV spectrophotometer (Nanodrop, Thermo Scientific, USA). Total RNA $(2 \mu \mathrm{g})$ was reverse transcribed into cDNA in a total volume of $15 \mu \mathrm{L}$ using the 
Reverse Transcriptase M-MLV(RNase H-) (Takara)according to the manufacturer's protocol. Each miRNA cDNA (10 ng) was obtained using the TaqMan Micro RNA Reverse Transcription Kit (Applied Bio systems) with the miRNA being reverse transcribed from the target miRNA using TaqMan Micro RNA Assays.

\subsection{Quantification of miRNA Expression}

miRNA expression was measured using real-time RT-PCR on the Applied Biosystems 7300 Real-Time PCR System (Applied Biosystems). U6 (Assay ID: 001973, Applied Biosystems) was used for internal control. Realtime PCR assay for miR-31 (Assay ID: 000185, Applied Biosystems) was used according to the manufacturer's instructions. Real-time conditions: $50^{\circ} \mathrm{C}$ for $2 \mathrm{~min}, 95^{\circ} \mathrm{C}$ for $10 \mathrm{~min}, 40$ cycles of $95^{\circ} \mathrm{C}$ for $15 \mathrm{sec}, 60^{\circ} \mathrm{C}$ for 1 min on an Applied Biosystems 7300 thermocycler (Applied Biosystems). All samples were run in duplicates.

In addition, real-time PCR analyses for Hb-9, Nestin and Stmn1were carried out using a SYBR Green PCR Kit (Invitrogen SYBR ${ }^{\circledR}$ Select Master Mix). All experiments were performed in duplicate. Copy numbers of cDNA for Hb-9, Nestin and Stmn1 were standardized to those of Rpl-19 for the same sample. Primer sequences for Hb-9 were as follows: 5'-CGAGACTCAGGTGAAGATTTGGT-3' (forward) and 5'-CTGCTCTTTGGCCTTTTTGC-3' (reverse); and for Stmn1, 5'-AGAAGCCGATGTAGGACCGTATAG-3' (forward) and 5'TCCCCTTGAGCCCCTAAAA-3'(reverse); and for Nestin, 5'-GGTCACTGTCGCCGCTACTC-3' (forward) and 5'-AAGCGGACGTGGAGCACTA-3' (reverse); and for Rpl-19, 5'-ATCCGCAAGCCTGTGACTGT-3' (forward) and 5'-TCGGGCCAGGGTGTTTTT-3' (reverse).

\section{Result}

\subsection{Isolation and Identification of NSCs}

After cultured 7 - 10 days, it can be observed number of neurospheres and volume was increased (Figure 1). Immunocytochemistry of Nestin display that the green fluorescence could be observed in spheres (Figure 2).

\subsection{Transfection of NSCs}

When transfection was succeed the positive cells was observed red fluorescence by fluorescence microscope. Five fields were randomly selected from slide to count positive cells and total cell numbers to calculate transfection efficiency. After 24 h, $48 \mathrm{~h}$ and 72 h, the transfection efficiency was 20\% - 30\%, 40\% - 55\% and 60\% $75 \%$, respectively (Figure 3(E)).

After one day, the cells added differentiation solution were observed a large number of death.

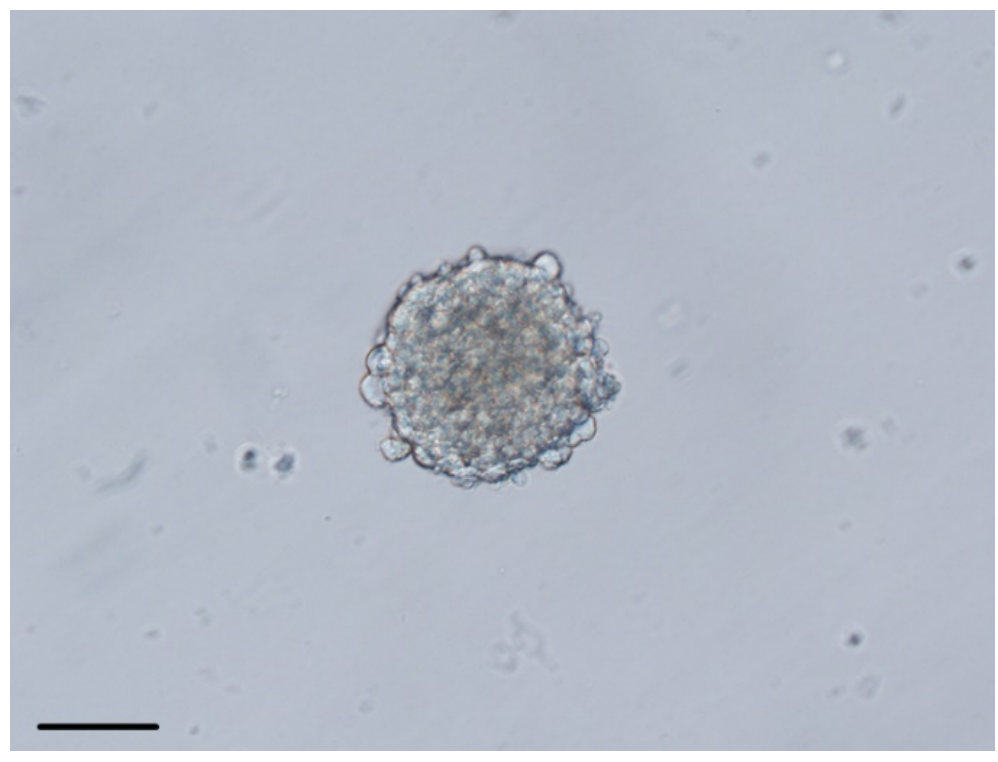

Figure 1. Observed under the light microscope, visible spherical neural stem cells, refraction and strong, clear boundaries. Scale bar $=50 \mu \mathrm{m}$. 

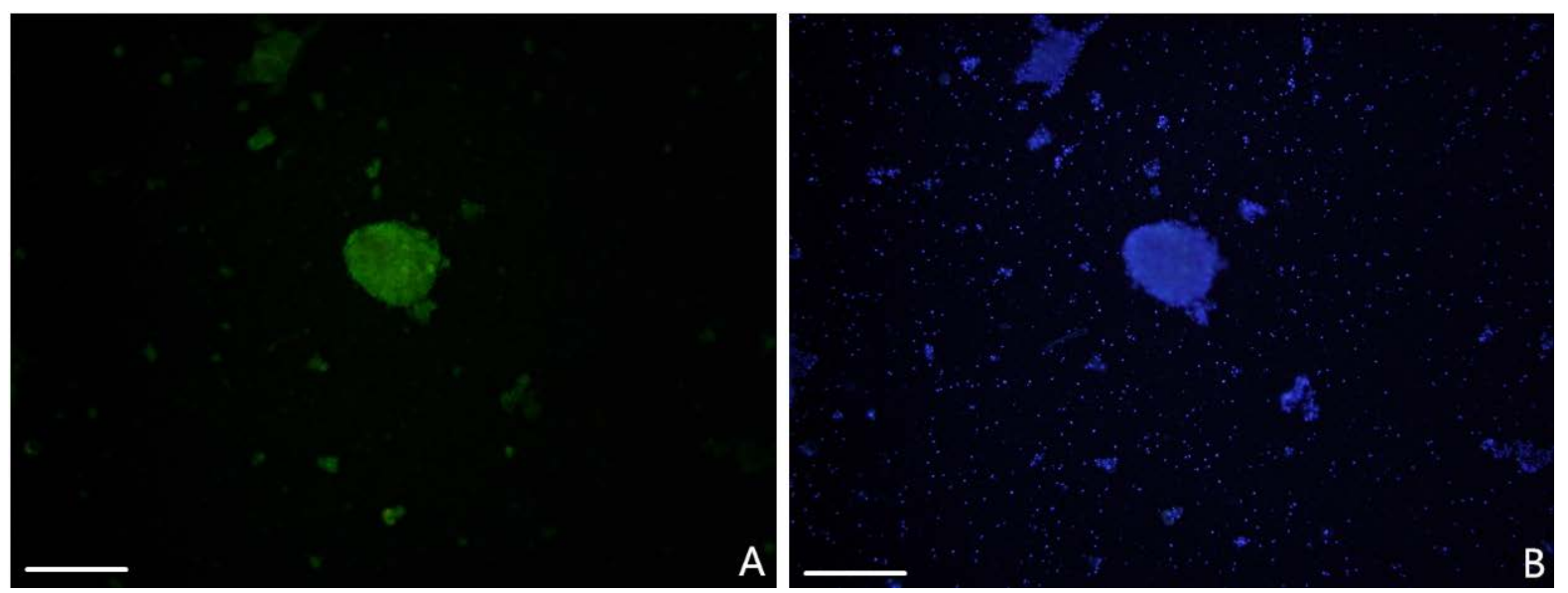

Figure 2. The photos of neural stem cell identification. (A) Immunofluorescent staining indicated that Nestin was stained in the cytoplasm with a clear nuclear boundary. Scale bar $=100 \mu \mathrm{m}$. (B) Immunofluorescent staining of Hochest indicated that the blue areas were nucleus. Scale bar $=100 \mu \mathrm{m}$.
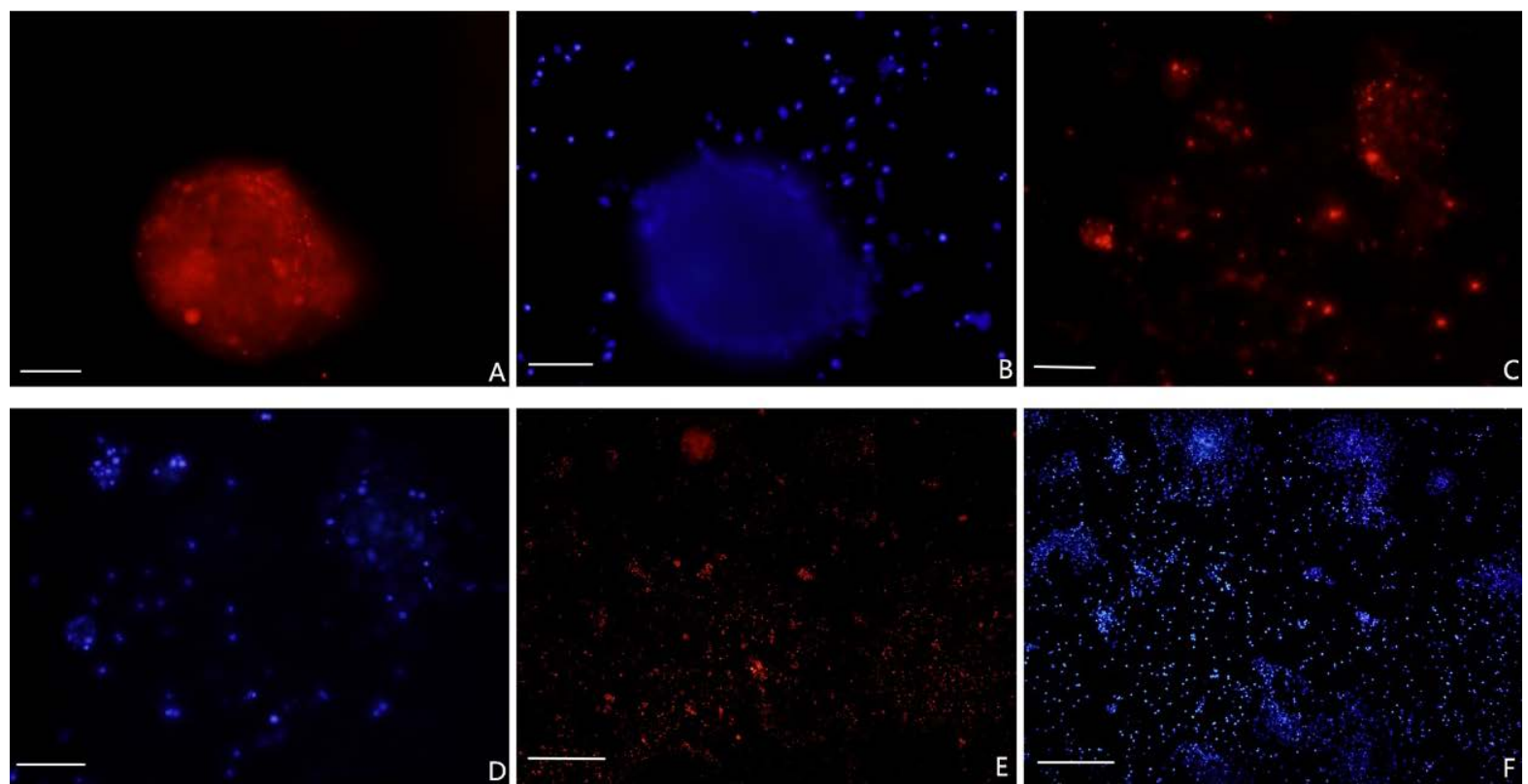

Figure 3. The transfection efficiency of RNAiMAX. As we can see in the photo, this effect will last with the time going on. (A) and (B) The first day, only a few of cells transfected successfully. Scale bar $=50 \mu \mathrm{m}$. (C) and (D) The second day, more cells was transfected successfully. Scale bar $=50 \mu \mathrm{m}$. (E) and (F) At the third day the efficiency is the highest. Scale bar $=$ $100 \mu \mathrm{m}$.

\subsection{Expression of ChAT}

After miR-31 were induced to differentiate into NSCs, ChAT stain was employed. The red fluorescence was seen under fluorescent microscope was ChAT positive cells. Compared with each group, the inhibitor group can emit stronger fluorescence (Figure $4(B)$ ), and the mimics group was significantly lower than inhibitor group (Figure 4(A)).

\subsection{Expression of miRNA and mRNA}

In Figure 5, RT-PCR assessment showed that expression of interference group was 0.006 and the interference control group was 0.181 higher than interference group; the highest is over-expression group, the expression was 21.081 higher than the over-expression control group was 0.726 . 

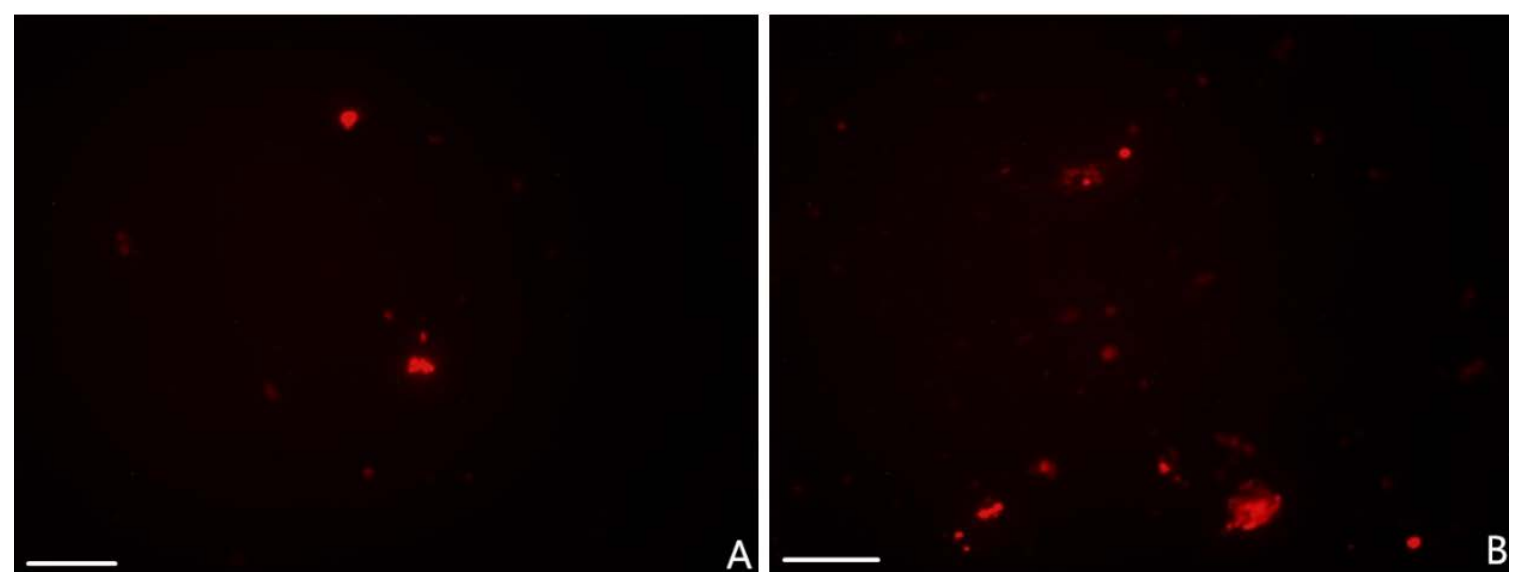

Figure 4. ChAT immunocytochemical identification. (A) There was weak positive expression in the mimics group. (B) A strongly positive expression was shown in the inhibitor group. Scale bar $=100 \mu \mathrm{m}$.

\section{miR-31}

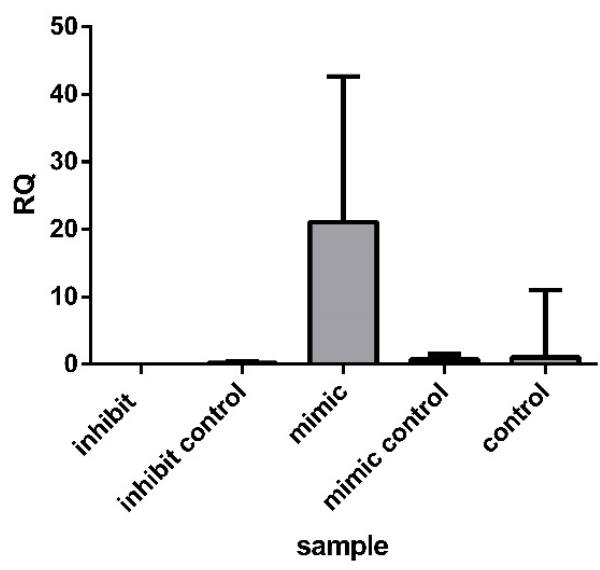

Figure 5. The results of induction efficiency. Compared with control group, the expression of miR-31 in mimics group was 0.006, and the expression of miR-31 in inhibitor group was 21.081 .

As shown in the Figure 6, the expression of genes were different among groups. In interference group, hb9 and stmn1 were up-regulated and Nestin was down-regulated. In over-expression group, Nestin, hb9 and stmn1 were up-regulated.

\section{Discussion}

MicroRNAs play an important role in gene regulated, it binds to complementary sites specifically on the 3'-untranslated regions of the target mRNAs to induce cleavage or repression of translation. In this way, miR-31 regulated protein synthesis to impress protein functional expression. It can be participated in many signaling pathway to control the development process of the individual and the physiological and pathological process. There are many studies about miRNA already, but their specific roles in physiological process are exploring.

Stem cell therapy is currently one of the promising approaches as many studies with a strong potential to promote functional recovery after SCI. It was being reported that transplantation therapy of stem cells has very big effect for spinal cord injury mouse. In our previous study, we found that injected NSCs from spinal to lesion location and it will promote the recovery, BBB score of treatment group obviously surpassed to the injury group. How to make more cells differentiated into neurons that had a positive effect on the functional outcome is the vital problem. Previous studies have shown that the level of miR-31 expression in NSCs was much higher than that in MNs. It also had been reported that miR-31 is an embryonic stem cell specific microRNA. So, miR-31 may be play an important role in maintaining NSCs in an undifferentiated state. 


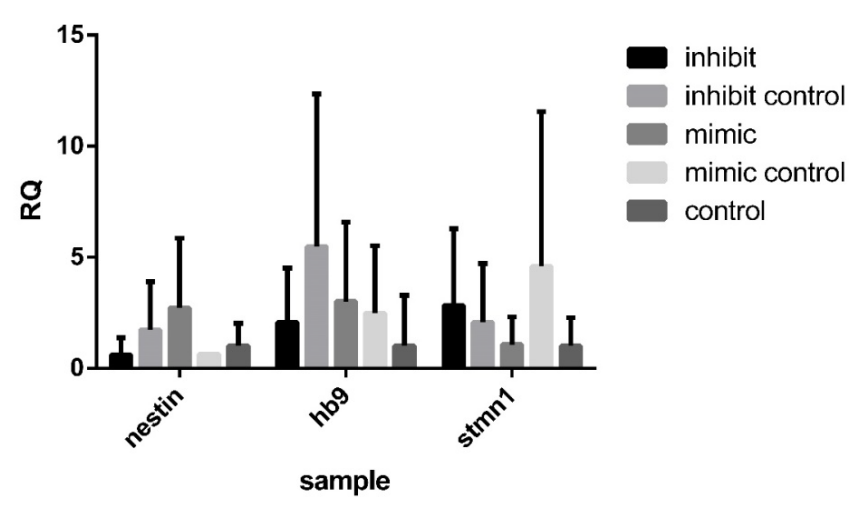

Figure 6. The results of relative gene expression.

So far, it is well accepted that quantity research of miR-31 related to cancer. Compared with normal tissues, miR-31 up-regulated at head and neck cancer (Wong et al, 2008) [7], colorectal cancer (motoyama et al, 2009; wang CJ et al, 2009) [8] [9], lung cancer (Liu X et al, 2010) [10] and so on; and down-regulated at breast cancer (Valastyan S et al, 2009) [11], prostatic cancer (Schaefer A et al, 2010) [12], gastric cancer (Guo J et al, 2009; Zhang Y et al, 2009) [13] [14]. Although there are a lot of research about miR-31, most of them are from the study of the process of disease. miRNA regulated signaling pathway to affect a series of protein in a biological processes. However, the effect that signaling pathways of miR-31 have yet to be fully defined.

The NSCs from spinal cord is a kind of NSCs, but its homology is better than other NSCs. Cell fate decisions can be regulated, so we hope that by regulate the expression of miR-31 in the spinal cord make the NSCs proliferation instead of NSCs injection treatment after spinal cord injury. We picked Nestin as the biomarker of NSCs and high expression at motor neurons hb9 and Stmn1. The goal of the study is to detect the expression of Nestin, hb9 and stmn1 after NSCs transfected miR-31 mimics, then we analyzed to try to explain the function of miR-31 in regulating NSCs functions in vitro.

Nestin is an intermediate filament protein, and it is expressed in dividing cells during the early stages of development in the CNS and peripheral nervous system. Therefore, it is widely used as a neuronal stem cell marker. It has been reported that the Nestin knockout mice were significantly smaller than their littermates, most of them will die at around 3 weeks after birth, and the survivor was sterile. Furthermore, a fraction of Nes-/-embryos already dead after E8.5 because neural tube defects.(Park D, 2010) [15] In our study, we found that the expression level of Nestin increased 2.72 times compared with control group after transfected miR-31 mimics, and it down-regulated 0.609 times when we transfected miR-31 inhibited. It illustrate that cells closer to stem cell state when miR-31 is overexpression.

Stathmin 1 (STMN1) is a microtubule-depolymerizing molecule that possess the capacity to bind tubulin and interfere with microtubule dynamics. It can take part in the process of proliferation and differentiation of motor neurons. (Yamada K, 2010) [16]. Sylvie Ozon was proved that STMN1 also participate in development of central nervous system. (Ozon S, 2002) [17] From this research we are aware that stmn1 was up-regulate 2.831 times when we inhibited miR-31, and it demonstrate that NSCs will differentiate into motor neurons when miR-31 was inhibited. Mohamed proved that STMN1 is a potentially target gene, over expression of miR-31 in KF-TX cells will reduced STMN1 expression. But in our experiment, STMN1 was no significant changes when miR-31 was over expression. (Hassan MK, 2015) [18].

The homeobox gene Hb9 is expressed selectively by motor neurons in the developing vertebrate CNS. But in our study, its expressions all up-regulated weather we transfected mimics or inhibit. What gives rise to this situation change has been an outstanding issue.

We herein investigated the role of miR-31 in the differentiation of NSCs. By controlling expression of miR-31, we found that it is consistent miR-31 maintaining NSCs in an undifferentiated state. Meantime, there are some different also. However, further studies are needed to explain the changes. Firstly, we will take western blot to test the protein level of related genes to determine the effect of miR-31. Secondly, we will detect the expression of other genes continually to study the signaling pathway of miR-31. The current studies of miRNAs signal pathways have also made some progress, a variety of signaling pathways are regulated by one miRNA. Therefore, we may use the mediating role of signaling pathways of miRNAs to guide cell differentiation, pro- 
vide a new reference for the treatment of diseases.

\section{Acknowledgements}

The study was supported by a grant from the The National Natural Science Foundation of China (81371384) and by the Experimental Animals Special Fundation in Shanxi Province (2014k15, 2014k06).

\section{References}

[1] Lu, P., Woodruff, G., Wang, Y.Z., Hunt, L.G.M., Wu, D., Boehle, E., Ahmad, R., Poplawski, G., Brock, J., Goldstein, L.S.B. and Tuszynski, M.H. (2014) Long-Distance Axonal Growth from Human Induced Pluripotent Stem Cells after Spinal Cord Injury. Neuron, 83, 789-796. http://dx.doi.org/10.1016/j.neuron.2014.07.014

[2] Suzuki, S.O. and Goldman, J.E. (2003) Multiple Cell Populations in the Early Postnatal Subventricular Zone Take Distinct Migratory Pathways: A Dynamic Study of Glial and Neuronal Progenitor Migration. J Neurosci, 23, 4240-4250.

[3] Harraz, M.M., Xu, J.-C. and Guiberson, N. (2014) miR-223 Regulates the Differentiation of Immature Neurons. Mol Cell Ther., 2, 1-15. http://dx.doi.org/10.1186/2052-8426-2-18

[4] Niu, C.S., Yang, Y. and Cheng, C.-D. (2013) miR-134 Regulates the Proliferation and Invasion of Glioblastoma Cells by Reducing Nanog Expression. 42, 1533-1540.

[5] Mor, E., Kano, S.-I., Colantuoni, C., Sawa, A., et al. (2013) microRNA-382 Expression Is Elevated in the Olfactory Neruoepithelium of Schizophrenia Patients. 55, 1-10.

[6] Wei, H.E., Wang, C.F., Zhang, C.S., Li, P.F., Wang, F. and Zhang, Z.Y. (2010) Comparative Profiling of microRNA Expression between Neural Stem Cells and Motor Neurons in Embryonic Spinal Cord in Rat. Int. J. Devl Neuroscience, 28, 545-551. http://dx.doi.org/10.1016/j.ijdevneu.2010.04.007

[7] Wong, T.S., Liu, X.B., Wong, B.Y., Ng, R.W., Yuen, A.P. and Wei, W.L. (2008) MaturemiR-184 as Potential Oncogenic microRNA of Squamous Cell Carcinoma of Tongue. Clin Cancer Res, 14, 2588-2592. http://dx.doi.org/10.1158/1078-0432.CCR-07-0666

[8] Motoyama, K., Inoue, H., Takatsuno, Y., Tanaka, F., Mimori, K., Uetake, H., Sugihara, K. and Mori, M. (2009) Overand Under-Expressed microRNAs in Human Colorectal Cancer. Int J Oncol, 34, 1069-1075.

[9] Wang, C.J., Zhou, Z.G., Wang, L., Yang, L., Zhou, B., Gu, J., Chen, H.Y. and Sun, X.F. (2009) Clinicopathological Significance of microRNA-31, -143 and -145 Expression in Colorectal Cancer. Dis Markers, 26, 27-34. http://dx.doi.org/10.1155/2009/921907

[10] Liu, X., Sempere, L.F., Ouyang, H., Memoli, V.A., Andrew, A.S., Luo, Y., Demidenko, E., Korc, M., Shi, W., Preis, M., Dragnev, K.H., Li, H., Direnzo, J., Bak, M., Freemantle, S.J., Kauppinen, S. and Dmitrovsky, E. (2010) MicroRNA-31 Functions as an Oncogenic microRNA in Mouse and Human Lung Cancer Cells by Repressing Specific Tumor Suppressors. J Clin Invest, 120, 1298-1309. http://dx.doi.org/10.1172/JCI39566

[11] Valastyan, S., Benaich, N., Chang, A., Reinhardt, F. and Weinberg, R.A. (2009) Concomitant Suppression of Three Target Genes Can Explain the Impact of a microRNA on Metastasis. Genes Dev., 23, 2592-2597. http://dx.doi.org/10.1101/gad.1832709

[12] Schaefer, A., Jung, M., Mollenkopf, H.J., Wagner, I., Stephan, C., Jentzmik, F., Miller, K., Lein, M., Kristiansen, G. and Jung, K. (2010) Diagnostic and Prognostic Implications of microRNA Profiling in Prostate Carcinoma. Int J Cancer, 126, 1166-1176.

[13] Guo, J., Miao, Y., Xiao, B., Huan, R., Jiang, Z., Meng, D. and Wang, Y. (2009) Differential Expression of microRNA Species in Human Gastric Cancer Versus Non-Tumorous Tissues. J Gastroenterol Hepatol, 24, 652-657. http://dx.doi.org/10.1111/j.1440-1746.2008.05666.X

[14] Zhang, Y., Guo, J., Li, D., Xiao, B., Miao, Y., Jiang, Z. and Zhuo, H. (2010) Down-Regulation of miR-31 Expression in Gastric Cancer Tissues and Its Clinical Significance. Med Oncol, 27, 685-689. http://dx.doi.org/10.1007/s12032-009-9269-x

[15] Park, D., Xiang, A.P., Mao, F.F., Zhang, L., Di, C.G., Liu, X.M., Shao, Y., Ma, B.F., Lee, J.H., Ha, K.S., Walton, N. and Lahn, B.T. (2010) Nestin Is Required for the Proper Self-Renewal of Neural Stem Cells. Stem Cells, 28, 21622171. http://dx.doi.org/10.1002/stem.541

[16] Yamada, K., Matsuzaki, S., Hattori, T., Kuwahara, R., Taniguchi, M., Hashimoto, H., Shintani, N., Baba, A., Kumamoto, N., Yamada, K., Yoshikawa, T., Katayama, T. and Tohyama, M. (2010) Increased Stathmin1 Expression in the Dentate Gyrus of Mice Causes Abnormal Axonal Arborizations. PLoS One, 5, e8596. http://dx.doi.org/10.1371/journal.pone.0008596

[17] Ozon, S., Guichet, A., Gavet, O., Roth, S. and Sobel, A. (2002) Drosophila Stathmin: A Microtubule-Destabilizing Factor 
Involved in Nervous System Formation. Mol Biol Cell, 13, 698-710. http://dx.doi.org/10.1091/mbc.01-07-0362

[18] Hassan, M.K., Watari, H., Mitamura, T., Mohamed, Z., El-Khamisy, S.F., Ohba, Y. and Sakuragi, N. (2015) P18/Stathmin1 Is Regulated by miR-31 in Ovarian Cancer in Response to Taxane. Oncoscience, 2, 294-308.

http://dx.doi.org/10.18632/oncoscience.143 